\title{
Cardiac shock wave therapy and myocardial perfusion in severe coronary artery disease
}

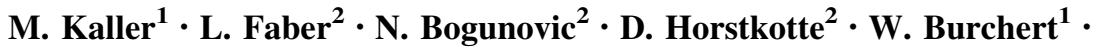 \\ Oliver Lindner ${ }^{1}$
}

Received: 28 January 2015/ Accepted: 31 March 2015/Published online: 17 April 2015

(c) The Author(s) 2015. This article is published with open access at Springerlink.com

\begin{abstract}
Background Ultrasound guided cardiac shock wave therapy (CSWT) is a noninvasive therapeutic option in the treatment of chronic-refractory angina. Clinical trials have shown that CSWT reduces angina symptoms, improves regional systolic function, LV ejection fraction, myocardial perfusion and quality of life parameters. Absolute measurements of myocardial perfusion before and after CSWT have not been performed so far.

Methods and results We studied a total of 21 CCS III patients with history of CAD and multiple interventions who suffered from disabling angina despite individually optimized medical therapy. An N-13 $\mathrm{NH}_{3}$ PET perfusion scan under adenosine was performed before and after CSWT treatment. CSWT was well tolerated in all patients. Absolute perfusion under adenosine of the global leftventricular myocardium did not change under therapy or minimal coronary resistance. The treated segments, however, showed in terms of both perfusion and resistance a mild but significant improvement, by 11 and $15 \%$, respectively, whereas no change could be observed in the remote segments. Considering a threshold of increased perfusion of $5 \%, 10(77 \%)$ out of 13 patients with a better target perfusion improved in their CCS class, whereas 3
\end{abstract}

Oliver Lindner

olindner@hdz-nrw.de

1 Institute of Radiology, Nuclear Medicine and Molecular Imaging, Heart and Diabetes Center North Rhine-Westphalia, University Hospital of the Ruhr-University Bochum, Georgstr. 11, 32545 Bad Oeynhausen, Germany

2 Clinic for Cardiology, Heart and Diabetes Center North Rhine-Westphalia, University Hospital of the RuhrUniversity Bochum, Bad Oeynhausen, Germany
(43\%) out of 7 patients without improved target perfusion improved in their CCS class too.

Conclusion Standard CSWT has the potential to improve myocardial perfusion of the therapy zone and clinical CAD symptomatology without affecting global myocardial perfusion. As a noninvasive and well tolerated therapeutic option, these data suggest the use of CSWT in patients with end-stage CAD.

Keywords Coronary artery disease $\cdot$ Cardiac shock wave therapy $\cdot$ Refractory angina $\cdot$ Myocardial perfusion imaging $\cdot$ PET

\section{Introduction}

Coronary artery disease (CAD) is one of the leading causes of morbidity and mortality in industrialized countries. Several therapeutic options for chronic CAD are available. They include medical treatment, percutaneous coronary intervention (PCI), and coronary artery bypass surgery. Despite excellent interventional results, an increasing number of patients with advanced CAD not suitable for any intervention suffer from chronic angina inspite of optimal medical therapy $[1,2]$. It has been shown that persistent angina symptoms are associated with long-term anxiety, depressions, impaired physical functioning and QOL [3]. Therefore, several alternative therapeutic approaches, including epidural spinal cord stimulation (SCS) [4], enhanced external counterpulsation (EECP) [5], transmyocardial laser revascularization (TMR) [6], or induction of angiogenesis by protein, gene stem cell-based therapies [7] have been proposed. These alternatives are invasive, still at a preclinical stage, or already outdated. 
Ultrasound guided cardiac shock wave therapy (CSWT) is another noninvasive therapeutic option in the treatment of chronic-refractory angina. Physically, a shock wave pulse is a single sharp pressure pulse with amplitude up to $100 \mathrm{MPa}$, followed by a tensile part of several microseconds with lower amplitude.

Animal studies have suggested that CSWT promoted angiogenesis in ischemic myocardium by mRNA expression of vascular endothelial growth factor (VEGF), endothelial cell proliferation, and endothelial nitric oxide synthase (eNOS) expression [8, 9]. Furthermore, nonenzymatic nitric oxide production by CSWT could be demonstrated [10].

Clinical trials demonstrated that CSWT may reduce angina symptoms, improve regional systolic function, LV ejection fraction, myocardial blood flow and quality of life parameters [11-14].

In this context myocardial perfusion has been evaluated with SPECT imaging at rest [15], under low-dose dobutamin stimulation [11, 13], on exercise [16] and under dipyridamole-induced hyperemia [17], all without absolute quantification. The present study evaluated with $\mathrm{N}-13 \mathrm{NH}_{3}$ PET perfusion imaging under adenosine induced hyperemia the effects of CSWT treatment on absolute perfusion in the target region and in the entire left myocardium in patients with chronic-refractory angina.

\section{Methods}

\section{Patient population and study protocol}

We studied a total of 21 patients with history of CAD and multiple prior interventions (PCI, CABG, or both) who suffered from disabling angina despite individually optimized medical therapy (Table 1).

An N-13 $\mathrm{NH}_{3}$ PET scan at rest and under adenosine was performed for clinical reasons to reevaluate a repeat

Table 1 Patient characteristics

\begin{tabular}{|c|c|c|}
\hline Age & & $65 \pm 10$ years \\
\hline Gender & & $13 \mathrm{~m}, 8 \mathrm{f}$ \\
\hline Diabetes & & 11 patients \\
\hline Hypertension & & 17 patients \\
\hline $\begin{array}{l}\text { Cardiac } \\
\text { history }\end{array}$ & No. of patients & CAD status \\
\hline CAD & 21 & 1 with 1 -, 20 with 3 -vessel disease \\
\hline $\begin{array}{l}\text { Prior } \\
\text { infarction }\end{array}$ & 11 & 7 with 1,4 with $2 \mathrm{MI}$ \\
\hline Prior PCI & 16 & 5 with 1,6 with 2,5 with $\geq 3 \mathrm{PCI}$ \\
\hline Prior CABG & 19 & 15 with 1,4 with $2 \mathrm{CABG}$ \\
\hline
\end{tabular}

mechanical revascularization. If reintervention had been found inappropriate, the viable myocardial wall with the leading perfusion abnormality was identified for CSWT. The baseline PET scan was performed $21 \pm 36$ days before onset of therapy. A repeat PET scan was performed $43 \pm 27$ days after the last CSWT session for clinical reasons, to verify the therapeutic effect on regional perfusion and to decide upon continuation of therapy.

Canadian Cardiovascular Society (CCS) scale was assessed in all patients before and after therapy. Additionally, echocardiography and, if feasible, ergometry were performed.

Patients gave their written informed consent to CSWT and the clinical PET studies. The data analysis was approved by the local ethics committee of the Ruhr-University Bochum (Reg. No. 60/2013).

\section{Image acquisition and stress testing}

Patients were investigated with an ECAT-951 R scanner (CTI/Siemens Medical systems) and, after a change of the device, with a Biograph mCT (Siemens, Erlangen, Germany). Directly before the PET acquisition, a transmission scan was performed with an ECAT-951 R scanner and a low-dose $\mathrm{CT}$ with the Biograph $\mathrm{mCT}$ for attenuation correction.

Adenosine was infused intravenously at a constant rate of $0.14 \mathrm{mg} / \mathrm{kg} / \mathrm{min}$ over $6 \mathrm{~min}$. During the stress phase, heart rate and blood pressure were recorded every $2 \mathrm{~min}$, starting with the onset of the adenosine infusion until completion of after 6 min. Mean arterial blood pressure was calculated from the average values of all four time points and minimal coronary resistance as mean arterial blood pressure/global perfusion.

Two minutes after the onset of the adenosine infusion, on average $600 \mathrm{MBq} \mathrm{N}-13 \mathrm{NH}_{3}$ were injected as an intravenous bolus. Image acquisition over 15 min was started simultaneously with the bolus injection. A consecutive set of 20 frames was reconstructed for quantification of perfusion.

\section{Quantitative perfusion analysis}

Quantification of the N-13 $\mathrm{NH}_{3}$ scans was based on an irreversible two-compartment model [18]. Corrections for fractional blood volume, partial volume effects and spillover activity from left-ventricular blood pool to tissue were calculated as described elsewhere [19]. A validation of the model in humans had been performed by the argon inert gas technique beforehand [20]. The quantification procedure delivered 20-segment parametric polar maps of MBF. Segments with a fractional blood volume $>0.5$ were excluded from further analysis. Such large values are only 
explainable by spillover from the ventricle and mostly referred to the basal segments of the septum. Furthermore, segments with a resting $\mathrm{MBF}<50 \mathrm{~mL} / \mathrm{min} / 100 \mathrm{~g}$ were regarded as scarred and also excluded from the analysis. Global perfusion was calculated as the average of all myocardial segments [21].

The CSWT target zone was assigned to the anteroseptal wall in 8 cases, to the inferolateral wall in 6 , to the inferior wall in 6 , and to the apex in 1 case. The reference wall was defined as the remote wall opposite to the CSWT target zone. In the case of the apical CSWT target, the anterior wall served as the reference. For quantitative analysis, perfusion values of the segments of the parametric polarmaps representing either the target or the remote wall were averaged.

\section{Shock wave therapy device, procedure and protocol}

A detailed description with illustration of the device and the CSWT protocol has been given elsewhere [13]. In brief, shockwaves were applied with a commercially available shock wave generator (Cardiospec, Medispec, Gaitersburg, USA) under echocardiographic guidance. The energy flux density was adjustable between 0.03 and $0.2 \mathrm{~mJ} / \mathrm{mm}^{2}$, with a focus size of $8-9 \mathrm{~mm}$ and a length of $25 \mathrm{~mm}$ to ensure transmural coverage. The focus could be set up to a depth between 4 and $15 \mathrm{~cm}$. During the procedure, patients lay in supine position. ECG, blood pressure and vital signs were monitored continuously.

The target region previously defined with the PET scan was adjusted with the ultrasound probe. Shock wave release occurred with an R-wave ECG trigger.

The CSWT scheme was performed over 3 months (93 \pm 34 days) in blocks of 3 sessions applied every other day during the treatment weeks, with 6 weeks intervals (44 \pm 25 and $42 \pm 15$ days, respectively). The target region previously defined with PET was divided into a basal, midcavity, and apical zone. During each treatment, these zones were targeted with the ultrasound probe, progressing from the basal zone in week 1 (sessions 1-3) to the midcavity zone in week 2 (sessions 4-6), and the apical zone in week 3 (sessions 7-9). Patients received 300 shocks (when starting) to 500 shocks (after verification of tolerance) per treatment session. Thus, during the 9 shockwave sessions, a total of 2700-4500 individual discharges were delivered. Troponin I was controlled several hours after each application.

\section{Echocardiography}

Resting two-dimensional echocardiography was performed at baseline and follow-up with a $1.7 / 3.3 \mathrm{MHz}$ multifrequency probe in harmonic imaging mode using commercially available echo equipment (Vivid E9, General Electric, Horten, Norway). The classical two apical views (2- and 4-chamber views) were applied to measure biplane LVEF and both the endsystolic and enddiastolic volumes.

\section{Exercise stress testing}

The bicycle test was performed in the upright position (Ergometer 900 ERG, GE Medical Systems). Depending on the patient's condition, ergometry was started at $25 \mathrm{~W}$ or $50 \mathrm{~W}$, and was increased by $25 \mathrm{~W}$ each minute. The test was stopped when typical chest pain, significant ST depressions, or exhaustion of the leg muscles occurred.

\section{Statistical analysis}

For the sample size estimation, a power of $90 \%$ and a significance criterion of 0.05 were chosen. The minimum expected difference between the two means and the standard deviation were estimated to $10 \mathrm{~mL} / \mathrm{min} / 100 \mathrm{~g}$ each. Accordingly, about 20 patients had to be enrolled [22].

Data are given as mean value \pm standard deviation. In the first step, the paired parameters were tested for normal distribution with the Kolmogorov-Smirnov test. As all parameters were normally distributed, post hoc comparisons were performed with a paired $t$ test.

The contingency between change in CCS class (unchanged or better) and change in perfusion (increase $<5 \%$ and increase $\geq 5$ ) was examined using Fisher's exact test.

Differences were considered statistically significant at values $<0.05$ (two-sided). For the analyses, the statistical software package IBM SPSS (version 20) was used.

\section{Results}

\section{Clinical characteristics, medication, and clinical parameters}

The baseline clinical characteristics of the patients and their cardiac medication are shown in Tables 1 and 2 . Neither dosages of long-acting nitrates nor the other cardiac medicaments were changed during the observation period.

CSWT was well tolerated in all patients. No patient showed an increase in troponin I beyond the reference level after the applications, and no therapy course had to be stopped prematurely.

All patients were in CCS class III at baseline. At followup, a downstaging was observed in 13 patients, such that 13 were in CCS class II and 8 remained in CCS class III (Table 3). 
Exercise testing could be completed in 16 patients at baseline and follow-up. In 5 patients, ergometry was not feasible due to non-cardiac limitations. Mean achievable workload at baseline was $93 \pm 44 \mathrm{~W}$, and at follow-up $101 \pm 41 \mathrm{~W}$ (n.s.).

\section{PET perfusion measurements and correlation to $\mathrm{CCS}$ class}

The perfusion data are listed in Tables 3 and 4. Absolute myocardial perfusion of the global left-ventricular

Table 2 List of cardiac medication

\begin{tabular}{lc}
\hline & No. of patients \\
\hline Aspirin & 14 \\
Clopidogrel & 7 \\
B-blockers & 21 \\
ACE inhibitors/AT1-receptor antagonists & 19 \\
Diuretics & 13 \\
Calcium antagonists & 4 \\
Nitrates & 21 \\
Ranolazin & 4 \\
Statins & 19 \\
\hline
\end{tabular}

myocardium under adenosine induced hyperemia did not change under therapy, nor did minimal coronary resistance (perfusion related to perfusion). The treated segments, however, in terms of both perfusion and resistance, showed a mild but significant improvement, 11 and $15 \%$, respectively, whereas, no change could be observed in the remote segments. Figure 1 illustrates the PET scans of a typical case. This patient experienced a downstaging to CCS class II.

Considering a threshold of improvement in perfusion of $5 \%$, based on an estimated error of measurement of $5 \%$, $10(77 \%)$ patients out of 13 with a better perfusion of the target segments improved in their CCS class, whereas, 3 (43\%) patients out of 7 without better perfusion of the target segments improved in their CCS class, too (Table 5). This $5 \%$ value was based on the general variability of repeated PET N-13 ammonia measurements under adenosine [23].

\section{Echocardiographic measurements}

The results are given in Table 6. No significant changes in the global left-ventricular parameters enddiastolic diameter (LVEDD), enddiastolic (LVEDV), endsystolic (LVESV)

Table 3 Target region, PET perfusion, and change in CCS class of the individual patients

\begin{tabular}{|c|c|c|c|c|c|c|c|c|}
\hline \multirow[t]{2}{*}{ Pat No. } & \multirow[t]{2}{*}{ CSWT Target } & \multicolumn{2}{|c|}{ Perfusion of target segments } & \multicolumn{2}{|c|}{ Perfusion of remote segments } & \multicolumn{2}{|c|}{ Perfusion of the global myocardium } & \multirow[t]{2}{*}{ Delta $\mathrm{CCS}^{\mathrm{a}}$} \\
\hline & & Baseline & Follow-up & Baseline & Follow-up & Baseline & Follow-up & \\
\hline 1 & Inferior & 91 & 123 & 131 & 150 & 130 & 156 & -1 \\
\hline 2 & Anteroseptal & 143 & 152 & 97 & 104 & 139 & 149 & 0 \\
\hline 3 & Lateral & 66 & 74 & 116 & 138 & 88 & 106 & -1 \\
\hline 4 & Anteroseptal & 87 & 71 & 141 & 107 & 105 & 75 & 0 \\
\hline 5 & Inferior & 184 & 162 & 244 & 171 & 241 & 192 & -1 \\
\hline 6 & Inferior & 114 & 156 & 174 & 209 & 144 & 171 & 0 \\
\hline 7 & Anteroseptal & 162 & 173 & 167 & 174 & 162 & 180 & 0 \\
\hline 8 & Lateral & 67 & 146 & 123 & 154 & 101 & 152 & -1 \\
\hline 9 & Lateral & 192 & 220 & 152 & 192 & 170 & 192 & -1 \\
\hline 10 & Lateral & 77 & 81 & 106 & 96 & 99 & 94 & -1 \\
\hline 11 & Apikal & 230 & 227 & 326 & 311 & 284 & 302 & 0 \\
\hline 12 & Anteroseptal & 116 & 130 & 90 & 96 & 96 & 95 & -1 \\
\hline 13 & Lateral & 76 & 93 & 134 & 150 & 94 & 120 & -1 \\
\hline 14 & Anteroseptal & 161 & 165 & 218 & 231 & 190 & 208 & 0 \\
\hline 15 & Anteroseptal & 164 & 167 & 204 & 196 & 178 & 183 & 0 \\
\hline 16 & Inferior & 76 & 101 & 79 & 91 & 94 & 103 & -1 \\
\hline 17 & Lateral & 119 & 135 & 140 & 117 & 157 & 161 & -1 \\
\hline 18 & Anteroseptal & 72 & 95 & 60 & 70 & 68 & 71 & -1 \\
\hline 19 & Inferior & 190 & 182 & 215 & 231 & 228 & 213 & -1 \\
\hline 20 & Anteroseptal & 77 & 92 & 115 & 99 & 82 & 81 & 0 \\
\hline 21 & Inferior & 58 & 42 & 79 & 88 & 75 & 86 & -1 \\
\hline
\end{tabular}

PET perfusion values are given in $\mathrm{mL} / \mathrm{min} / 100 \mathrm{~g}$

a -1 : a change from CCS classes III to II was observed, 0: no change in CCS class 
Table 4 PET perfusion measurements of the entire cohort

\begin{tabular}{|c|c|c|c|c|c|c|}
\hline & \multicolumn{3}{|c|}{ Perfusion under adenosine $(\mathrm{mL} / \mathrm{min} / 100 \mathrm{~g})$} & \multicolumn{3}{|c|}{ Minimal coronary resistance $[\mathrm{mmHg} /(\mathrm{mL} / \mathrm{min} / 100 \mathrm{~g})]$} \\
\hline & Baseline & Follow-up & $P$ & Baseline & Follow-up & $P$ \\
\hline CSWT target segments & $120 \pm 52$ & $133 \pm 49$ & 0.014 & $0.84 \pm 0.36$ & $0.72 \pm 0.30$ & 0.028 \\
\hline Remote segments & $148 \pm 64$ & $151 \pm 61$ & 0.6 & $0.67 \pm 0.31$ & $0.66 \pm 0.26$ & 0.76 \\
\hline Global myocardium & $139 \pm 59$ & $147 \pm 59$ & 0.1 & $0.80 \pm 0.38$ & $0.71 \pm 0.33$ & 0.24 \\
\hline
\end{tabular}
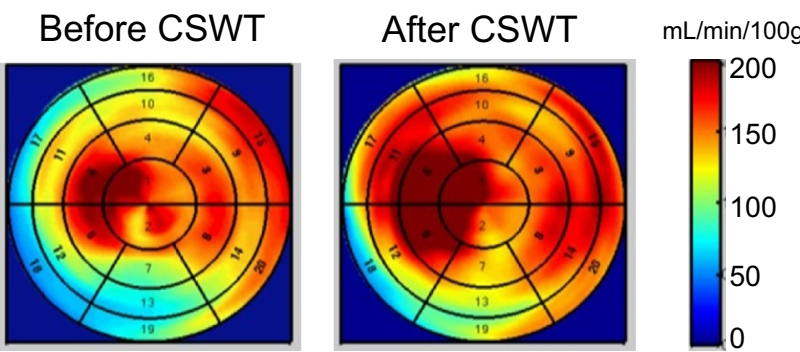

Fig. 1 PET polartomograms of absolute perfusion under adenosine of patient 1 (Table 3) before and after CSWT. In this case, the inferior wall was treated. Perfusion of the target zone under adenosine was 91 before CSWT and $123 \mathrm{~mL} / \mathrm{min} / 100 \mathrm{~g}$ after therapy

Table 5 CCS class and perfusion data

\begin{tabular}{llc}
\hline & CCS unchanged & CCS improved \\
\hline Perfusion shift $<5 \%$ & 5 & 3 \\
Perfusion shift $\geq 5 \%$ & 3 & 10 \\
\hline$P$
\end{tabular}

$P=0.16$

Table 6 Echocardiographic measurements

\begin{tabular}{llll}
\hline & Baseline & Follow-up & $P$ \\
\hline LVEDD $(\mathrm{mm})$ & $55 \pm 7$ & $53 \pm 6$ & 0.36 \\
LVEDV (ml) & $91 \pm 34$ & $96 \pm 25$ & 0.46 \\
$\operatorname{LVESV}(\mathrm{ml})$ & $47 \pm 33$ & $49 \pm 24$ & 0.29 \\
$\operatorname{LVEF}(\%)$ & $54 \pm 17$ & $51 \pm 14$ & 0.74 \\
\hline
\end{tabular}

LVEDD left-ventricular enddiastolic diameter, $L V E D V$ left-ventricular enddiastolic volume, $L V E S V$ left-ventricular endsystolic volume, $L V E F$ left-ventricular ejection fraction

and ejection fraction (LVEF) were observed between baseline and follow-up.

\section{Discussion}

CSWT considers an alternative therapeutic approach in the treatment of patients with end-stage CAD without any further interventional option, but with angina symptoms despite optimized individual medication. Although the exact mechanism of CSWT in myocardial tissue is not completely understood, the main effect seems to be a local stimulation of neoangiogenesis. This may be induced by two pathways: (1) increase of local shear stress by the collapse of microbubbles, and (2) stimulation of local angiogenesis by angiogenesis-related growth factors and recruitment of endothelial progenitor cells $[8,9,24]$.

Several studies demonstrated that CSWT is able to improve clinical symptoms, and quality of life parameters $[11,12,14]$. Evaluations have been performed with several nuclear imaging techniques. They showed, on a qualitative basis of imaging analysis, an improvement in resting perfusion in 14 patients and exercise perfusion in 10 patients $[15,16]$. A more dedicated analysis in 9 patients demonstrated an improvement in the treated area, while the untreated area tended to worsen [17]. One study failed to show significant differences in myocardial perfusion in 9 patients at rest and under low-dose dobutamine stimulation [11].

In the present study, we could demonstrate a significant increase in absolute myocardial perfusion in the treated segments, whereas no change in perfusion occurred in the remote segments. Coronary resistance showed a concordant behavior indicating that the perfusion changes were not exclusively related to improvements in hemodynamics and has to be assigned to further factors directly affecting myocardial perfusion. CSWT-induced neoangiogenesis may be one potential component, but the extent to which it contributes required additional morphological studies.

Normal absolute myocardial perfusion under hyperemia is usually assumed $>200 \mathrm{~mL} / \mathrm{min} / 100 \mathrm{~g}$ [25]. Against this background, mean global myocardial perfusion under adenosine was severely diminished in our patient cohort, but within the range of hyperemic perfusion in ischemic heart disease [26]. The relative high standard deviation of the individual data indicates that they extend over a long range and that the CSWT patient group itself represents a heterogeneous subset.

In terms of global myocardial perfusion and minimal coronary resistance, no significant changes were found during the 4 months between the observation periods. These results are in accordance with the echocardiographic measurements indicating that no significant change of the disease occurred during this period. Nevertheless, a treatment of the leading ischemic area seems to be beneficial 
with respect to the CCS classification, although no significant changes in exercise tolerance were observed. To what extent this improvement in CCS classification is a net effect of reduced ischemic burden, and thus a relief of angina symptoms, or is driven by changes in individual perception needs further clarification. The present data indicate that suggestive factors are likely to play a role as $38 \%$ of those patients with no better perfusion of the target segments experienced a CCS class downstaging.

Another issue of interest is the duration of the improvements, with the concept for future therapeutic courses having to evaluate the frequency and duration of CSWT and, finally, patient outcome.

The results of the present study are in line with former imaging studies and demonstrate absolute effects of CSWT on myocardial perfusion of the target zone [17]. The clinical results, however, are more cautious than those of other studies, but concordant to the global perfusion analysis which indicates that the underlying end-stage CAD remains as expected and that a local noninvasive procedure may not significantly improve an advanced stage of disease $[11,13]$.

To summarize the results of the present study: CSWT has the potential to improve myocardial perfusion of the target therapy zone and CAD symptomatology without affecting global myocardial perfusion and morphologic ventricular parameters. As a noninvasive and well tolerated therapeutic option, our data suggest the use of CSWT in patients with end-stage CAD.

\section{Study limitations}

From a statistical point of view, the patient number is sufficient to identify differences in perfusion with PET imaging and mildly larger than in other studies with nuclear medicine imaging. Nevertheless, larger patient cohorts with longer follow-ups are needed to further evaluate the efficacy of CSWT. A placebo-controlled group is missing. However, the intraindividual comparison of treated vs nontreated zones tried to circumnavigate this issue.

Acknowledgments The authors wish to thank Mrs. S. Kirkby for revision of the manuscript, and especially Prof. Dr. A. Zittermann for his helpful assistance in the statistical analysis.

\section{Conflict of interest None}

Open Access This article is distributed under the terms of the Creative Commons Attribution 4.0 International License (http:// creativecommons.org/licenses/by/4.0/), which permits unrestricted use, distribution, and reproduction in any medium, provided you give appropriate credit to the original author(s) and the source, provide a link to the Creative Commons license, and indicate if changes were made.

\section{References}

1. Jolicoeur EM, Granger CB, Henry TD et al (2008) Clinical and research issues regarding chronic advanced coronary artery disease: part I: contemporary and emerging therapies. Am Heart $\mathbf{J}$ 155(3):418-434

2. Jolicoeur EM, Ohman EM, Temple R et al (2008) Clinical and research issues regarding chronic advanced coronary artery disease part II: trial design, outcomes, and regulatory issues. Am Heart J 155(3):435-444

3. Jespersen L, Abildstrom SZ, Hvelplund A, Prescott E (2013) Persistent angina: highly prevalent and associated with long-term anxiety, depression, low physical functioning, and quality of life in stable angina pectoris. Clin Res Cardiol 102(8):571-581

4. Tronnier V, Baron R, Birklein F et al (2011) Epidural spinal cord stimulation for therapy of chronic pain. Summary of the S3 guidelines. Schmerz 25(5):484-492

5. Sharma U, Ramsey HK, Tak T (2013) The role of enhanced external counter pulsation therapy in clinical practice. Clin Med Res 11(4):226-232

6. Allen KB, Kelly J, Borkon AM et al (2008) Transmyocardial laser revascularization: from randomized trials to clinical practice. A review of techniques, evidence-based outcomes, and future directions. Anesthesiol Clin 26(3):501-519

7. Attanasio S, Schaer G (2011) Therapeutic angiogenesis for the management of refractory angina: current concepts. Cardiovasc Ther 29(6):e1-e11

8. Nishida T, Shimokawa H, Oi K et al (2004) Extracorporeal cardiac shock wave therapy markedly ameliorates ischemia-induced myocardial dysfunction in pigs in vivo. Circulation 110(19):3055-3061

9. Mariotto S, Cavalieri E, Amelio E et al (2005) Extracorporeal shock waves: from lithotripsy to anti-inflammatory action by NO production. Nitric Oxide 12(2):89-96

10. Gotte G, Amelio E, Russo S et al (2002) Short-time non-enzymatic nitric oxide synthesis from L-arginine and hydrogen peroxide induced by shock waves treatment. FEBS Lett 520(1-3):153-155

11. Wang Y, Guo T, Cai HY et al (2010) Cardiac shock wave therapy reduces angina and improves myocardial function in patients with refractory coronary artery disease. Clin Cardiol 33(11):693-699

12. Leibowitz D, Weiss AT, Rott D, Durst R, Lotan C (2013) The efficacy of cardiac shock wave therapy in the treatment of refractory angina: a pilot prospective, randomized, double-blind trial. Int J Cardiol 167(6):3033-3034

13. Zuoziene G, Laucevicius A, Leibowitz D (2012) Extracorporeal shockwave myocardial revascularization improves clinical symptoms and left ventricular function in patients with refractory angina. Coron Artery Dis 23(1):62-67

14. Schmid JP, Capoferri M, Wahl A, Eshtehardi P, Hess OM (2012) Cardiac shock wave therapy for chronic refractory angina pectoris. A prospective placebo-controlled randomized trial. Cardiovasc Ther 31(3): e1-e6

15. Yang P, Guo T, Wang W et al (2013) Randomized and doubleblind controlled clinical trial of extracorporeal cardiac shock wave therapy for coronary heart disease. Heart Vessels 28(3):284-291

16. Khattab AA, Brodersen B, Schuermann-Kuchenbrandt D et al (2007) Extracorporeal cardiac shock wave therapy: first experience in the everyday practice for treatment of chronic refractory angina pectoris. Int J Cardiol 121(1):84-85

17. Fukumoto Y, Ito A, Uwatoku $\mathrm{T}$ et al (2006) Extracorporeal cardiac shock wave therapy ameliorates myocardial ischemia in patients with severe coronary artery disease. Coron Artery Dis 17(1):63-70 
18. Hutchins GD, Schwaiger M, Rosenspire KC et al (1990) Noninvasive quantification of regional blood flow in the human heart using N-13 ammonia and dynamic positron emission tomographic imaging. J Am Coll Cardiol 15(5):1032-1042

19. van den Hoff J, Burchert W, Borner AR et al (2001) [1(11)C]Acetate as a quantitative perfusion tracer in myocardial PET. J Nucl Med 42(8):1174-1182

20. Kotzerke J, Glatting G, van den Hoff J et al (2001) Validation of myocardial blood flow estimation with nitrogen-13 ammonia PET by the argon inert gas technique in humans. Eur $\mathrm{J}$ Nucl Med 28(3):340-345

21. Hoffmeister C, Preuss R, Weise R, Burchert W, Lindner O (2014) The effect of beta blocker withdrawal on adenosine myocardial perfusion imaging. J Nucl Cardiol 21(6):1223-1229

22. Eng J (2003) Sample size estimation: how many individuals should be studied? Radiology 227(2):309-313
23. Sawada S, Muzik O, Beanlands RS et al (1995) Interobserver and interstudy variability of myocardial blood flow and flow-reserve measurements with nitrogen 13 ammonia-labeled positron emission tomography. J Nucl Cardiol 2(5):413-422

24. Aicher A, Heeschen C, Sasaki K et al (2006) Low-energy shock wave for enhancing recruitment of endothelial progenitor cells: a new modality to increase efficacy of cell therapy in chronic hind limb ischemia. Circulation 114(25):2823-2830

25. Sdringola S, Johnson NP, Kirkeeide RL, Cid E, Gould KL (2011) Impact of unexpected factors on quantitative myocardial perfusion and coronary flow reserve in young, asymptomatic volunteers. JACC Cardiovasc Imaging 4(4):402-412

26. Tio RA, Dabeshlim A, Siebelink HM et al (2009) Comparison between the prognostic value of left ventricular function and myocardial perfusion reserve in patients with ischemic heart disease. J Nucl Med 50(2):214-219 